\section{Medizinische Entscheidungen am Lebensende in der Schweiz}

Die rechtliche Regelung der Sterbehilfe steht in der Schweiz zur Zeit hoch oben auf der politischen Agenda. Die Schweizerische Akademie der Medizinischen Wissenschaften (SAMW) hat in ihrem Memorandum, welches sie im Dezember 1999 als Antwort auf den Bericht "Sterbehilfe" des EJPD erstellt hat, als einen der Kernpunkte auf die mangelnde Datenlage im Bereich der persönlichen Haltungen, Erfahrungen und Einstellungen der Ärzteschaft hingewiesen. Wir wissen nur wenig darüber, wie die Menschen in der Schweiz sterben und welche Haltung die Ärztinnen und Ärzte einnehmen, welche die Sterbenden begleiten.

In diesem Heft wird Ihnen eine Studie vorgestellt, welche zum Ziel hat, diese Wissenslücken zu füllen. Im Rahmen einer EU-Studie, an welcher die Schweiz als eines von sechs europäischen Ländern teilnimmt, sollen einerseits anhand von konkreten Todesfällen die medizinischen Entscheidungen am Lebensende eruiert werden, andererseits sollen Ärztinnen und Ärzte - ausgewählt anhand einer Zufallsstichprobe - anhand von Fragebogen über ihre persönlichen Einstellungen und Erfahrungen im Umgang mit sterbenden Menschen Auskunft geben.
Der Erfolg dieser Studie hängt ganz entscheidend von der Kooperation der Ärztinnen und Ärzte $a b$. Einen solchen Fragebogen auszufüllen bedingt einerseits ein Stück persönlichen Mut und andererseits ein grosses Vertrauen in die beteiligten Institutionen. Vertrauen darin, dass diese persönlichen Daten anonymisiert werden, so dass zu keinem Zeitpunkt irgendwelche Rückschlüsse auf Personen gezogen werden können. Die beiden verantwortlichen Institutionen, das Institut für Sozial- und Präventivmedizin sowie das Institut für Rechtsmedizin der Universität Zürich, setzen mit dem geplanten Studiendesign alles daran, dass Sie, liebe Kolleginnen und Kollegen, ihnen das Vertrauen schenken dürfen. Die SAMW stellt sich mit Überzeugung hinter diese Studie, da sie genau diejenigen wichtigen Daten hervorbringen wird, die essentiell sind für eine aufgeklärte und verantwortungsvolle Diskussion über die Regelung der Sterbehilfe in der Schweiz und die zur Zeit noch fehlen.

Wir bitten Sie deshalb, sich an dieser Studie aktiv zu beteiligen, falls Sie angeschrieben werden. Sie leisten damit einen wesentlichen Beitrag dafür, dass ein zukünftiger gesellschaftlicher und politischer Diskurs auf soliden Erkenntnissen beruht und damit ethisch vertretbar ist.

Die SAMW dankt Ihnen dafür.

Prof. Werner Stauffacher, Präsident SAMW 